\title{
Ultrastructural remodelling of slow skeletal muscle fibres in creatine kinase deficient mice: a quantitative study
}

\author{
Marta Novotová ${ }^{,}$Bohumila Tarabová ${ }^{1}$, Lucia Tylková ${ }^{1}$, Renée Ventura-Clapier ${ }^{2}$ and \\ Ivan Zahradník ${ }^{1}$ \\ ${ }^{1}$ Institute of Molecular Physiology and Genetics, Slovak Academy of Sciences, Bratislava, Slovak Republic \\ ${ }^{2}$ Signaling and cardiovascular pathophysiology UMR-S 1180, Inserm, Univ. Paris-Sud, Université Paris-Saclay, Châtenay- \\ Malabry, France
}

\begin{abstract}
Creatine kinase content, isoform distribution, and participation in energy transfer are muscle type specific. We analysed ultrastructural changes in slow muscle fibres of soleus due to invalidation of creatine kinase (CK) to reveal a difference in the remodelling strategy in comparison with fast muscle fibres of gastrocnemius published previously. We have employed the stereological method of vertical sections and electron microscopy of soleus muscles of wild type (WT) and $\mathrm{CK}^{-/-}$mice. The mitochondrial volume density was $1.4 \times$ higher but that of sarcoplasmic reticulum (SR) was almost $5 \times$ lower in slow $\mathrm{CK}^{-/-}$muscles fibres than in WT fibres. The volume density of terminal cisterns and of $\mathrm{t}$-tubules was also lower in $\mathrm{CK}^{-/-}$than in WT fibres. The analysis of organelle environment revealed increased neighbourhood of mitochondria and A-bands that resulted from the decreased volume density of SR, from relocation of mitochondria along myofibrils, and from intrusion of mitochondria to myofibrils. These processes direct ATP supply closer to the contractile machinery. The decreased interaction between mitochondria and SR suggests reduced dependence of calcium uptake on oxidative ATP production. In conclusion, the architecture of skeletal muscle cells is under control of a cellular program that optimizes energy utilization specifically for a given muscle type.
\end{abstract}

Key words: Skeletal muscle cells — Organelle environment - Adaptation — Energy deficiency

\section{Introduction}

The functional and structural diversity of skeletal muscles relies on the diversity of their muscle fibres that differ in morphological, biochemical and contractile properties. The high degree of molecular variability is due to the existence of multigenic protein families whose expression can respond to altered functional demands. The fast and slow skeletal muscles are composed of several muscle fibre types differing substantially in expression and protein content of myosin heavy chain isoforms that determine activity of myosin ATPase and contractile performance (Žurmanová and Soukup 2013). Schematically, two distinct metabolic patterns related to muscle phenotype were defined in mammals. The

Correspondence to: Ivan Zahradník, Institute of Molecular Physiology and Genetics, Slovak Academy of Sciences, Dúbravská cesta 9, P.O. Box 63, 84005 Bratislava, Slovak Republic

E-mail: ivan.zahradnik@savba.sk fast skeletal muscle fibres develop strong and fast contractions for short periods at the expense of their metabolic reserves. On the contrary, the slow muscle fibres develop lower contractile force and sustain long-term contractile activity during which they rely on oxidative phosphorylation. Both muscle cell types contain an energy transfer system based on creatine kinase (CK). The fast muscle cells rely on phosphocreatine $(\mathrm{PCr})$ as an energy reservoir and on $\mathrm{CK}$ reaction as a spatio-temporal buffer of adenine nucleotides (Meyer et al. 1984). The slow muscle cells depend on localization of CK isoenzymes that ensure efficient integration between energy utilization and production (for review see Wyss et al. 1992; Saks et al. 1994).

The importance of CK-catalysed reactions in cellular energy networks was assessed in mice deficient in either the mitochondrial $\left(\mathrm{mi}-\mathrm{CK}^{-/}\right)$, the cytosolic $\left(\mathrm{M}-\mathrm{CK}^{-/-}\right.$), or both $\left(\mathrm{CK}^{-/-}\right)$enzyme isoforms (van Deursen et al. 1993; Steeghs et al. 1997). These animals appeared apparently viable but their muscles exhibited changes in mitochondrial 
morphology and composition, presence of a hyperproliferative sarcoplasmic reticulum, altered $\mathrm{Ca}^{2+}$ response and impaired force generation (Steeghs et al. 1997; Watchko et al. 1997). These mice also showed altered myofibrillar function and changes in mitochondrial regulation (Veksler et al. 1995; Ventura-Clapier et al. 1995). A detailed electron microscopy study revealed remodelling of the mitochondrial network (Novotova et al. 2006). While in the fast wild-type fibres the mitochondria were present in small quantities, mostly under sarcolemma and near Z-lines, in the fast $\mathrm{CK}^{-/-}$muscle fibres the new mitochondria arranged near myosin filaments and longitudinal sarcoplasmic reticulum. Stereologic analysis provided quantitative data that helped to understand the contribution of the structural remodelling to functional adaptation in response to CK deficiency (Novotova et al. 2006). Increased volume density, and proportionally increased surface density, of mitochondria in $\mathrm{CK}^{-/-}$muscle defined the extent of increased oxidative capacity for production of ATP. Other identified significant changes included: 1) increased volume of terminal cisterns of sarcoplasmic reticulum, suggesting increased capacity for excitation-contraction coupling, 2) reduced volume of the A-band at constant surface, suggesting decreased diffusion distance for calcium and energy substrates, 3) changes in the environment of the intermyofibrillar mitochondria, providing clear evidence for translocation of mitochondria towards the A-band, that is, closer to regions of high ATP utilization (Novotova et al. 2006). In functional studies, remodelling of the cell ultrastructure and proliferation of mitochondria observed in CK-lacking fast fibres was associated with rescue of calcium uptake and myofibrillar function due to direct energetic interaction of mitochondria with myofibrils and sarcoplasmic reticulum (Kaasik et al. 2001).

It remained to be investigated whether structurally, functionally and metabolically different types of muscle fibres respond to deficient energy availability in similar way or follow their own program. In this study, we analysed ultrastructure of soleus muscle fibres in $\mathrm{CK}^{-/-}$mice to reveal specificities in remodelling of slow twitch fibres and to identify the adaptation program that optimizes energy utilization to retain contractile function in the absence of creatine kinase.

\section{Material and Methods}

\section{Animals}

Soleus muscles of adult male C57BL/6 mice (3-5 month old) were isolated from five wild type (WT) and five $\mathrm{CK}^{-/-}$mice (mixed genetic background: C57BL/6 × 129/Sv) with null mutation of the cytosolic MM-CK and the mitochondrial mi-CK creatine kinase genes. Engineered mice were a kind gift from Drs. B. Wieringa and F. Oerlemans (University of
Nijmegen, Nijmegen, The Netherlands). Animals were sacrificed by intraperitoneal injection of lethal dose of sodium thiopental $(150 \mathrm{mg} / \mathrm{kg})$ in accordance with the institutional guidelines defined by the European Community guiding principles in the care and use of animals and French decree no. $87 / 848$ of October 19,1987 . Authorization to perform animal experiments according to this decree was obtained from the French Ministry of Agriculture and Fisheries (no. 7474, May 2, 2002).

\section{Electron microscopy}

Dissected soleus muscles were mounted in the washing chamber at its resting length, then stretched by $30 \%$, perfused with $2 \%$ glutaraldehyde in the cacodylate buffer (in $\mathrm{mmol} / \mathrm{l}$ : 150 sodium cacodylate, $2.0 \mathrm{CaCl}_{2}, \mathrm{pH} 7.3$ ) and fixed for 45 minutes. Five sample cubes of about $2 \mathrm{~mm}$ sides were dissected at random from superficial regions around the central part of the muscles. Samples were then re-fixed for $30 \mathrm{~min}$ in $2 \%$ glutaraldehyde in cacodylate buffer, post-fixed by $1 \%$ osmium tetroxide in cacodylate buffer for $30 \mathrm{~min}$ and contrasted with $2 \%$ aqueous solution of uranyl acetate. After gradual dehydration in ethanol, the samples were embedded through acetone in Durcupan (Fluka, Geneva, Switzerland). Ultrathin 70-80 nm longitudinal sections were placed on 400 mesh copper grids covered with formvar, stained with lead citrate and studied using JEM 1200 (JEOL, Tokyo, Japan) electron microscope. Micrographs were taken on $6.5 \times 9 \mathrm{~cm}$ Scientia EM films (Agfa, Mortsel, Belgium).

\section{Stereologic analysis}

The stereologic analysis was performed as described in detail earlier (Novotova et al. 2006). Briefly, longitudinal sections of the tissue blocks were taken at 3 randomly selected levels separated by more than 50 microns. The central part of each section was photographed at magnification of $12000 \times$. Micrographs were digitized at $600 \mathrm{dpi}$ resolution, converted to positives and balanced uniformly by the contrast and brightness controls in Adobe Photoshop (version 6.0, Adobe systems).

The cycloidal test grid for the method of vertical sections (Baddeley et al. 1986) was generated using STESYS software (Karen et al. 1998). The test grid was composed of 532 test points separated by $257 \mathrm{~nm}$. The length of the cycloid was 400 $\mathrm{nm}$. We have evaluated a section area of $4026 \mu \mathrm{m}^{2}$ of WT fibres, and $3843 \mu \mathrm{m}^{2}$ of $\mathrm{CK}^{-/-}$fibres. Volume and surface densities of the organelles, environment of mitochondria and myofibrils were estimated according to Novotova et al. (2006).

\section{Statistical analysis}

The statistical analysis was performed using Origin (version 7.0; OriginLab Co., USA). The data are expressed as mean 
\pm SE. Student's t-test was used to determine the statistical significance of differences between group means. Statistical significance was defined at $p<0.05$.

\section{Results}

\section{Morphologic analysis}

In longitudinal sections, slow muscle fibres of wild type soleus (Fig. 1A) were characterized by rows of densely packed myofibrils. Sarcomeres were slightly out of register due to small shift of myofibrils relative to each other. Mitochondria were regular in shapes. Subsarcolemmal mitochondria were either distributed evenly at sides of I-bands and A-bands or clustered near nuclei. Intermyofibrillar mitochondria were localized between myofibrils mostly near I-bands. Sarcoplasmic reticulum was abundant near myofibrils and mitochondria, typically separating them from each other (Fig. 1A). Terminal cisterns of sarcoplasmic reticulum and t-tubules of sarcolemma were transversal to the long axis of the fibre close to I-band/A-band borders.
Ultrastructure of soleus muscle fibres of $\mathrm{CK}^{-/-}$mice was less homogenous than that of the WT ones. In addition to regions rather similar to that of WT fibres, like the myofibrillar region in Fig. 1B, regions with various irregularities were observed. Mitochondria of both the subsarcolemmal and intermyofibrillar populations varied in shape and size (Fig. 1B, 2A). Individual mitochondria expanded sometimes over more than one sarcomere. Mitochondria were packed closely to myofibrils sometimes visible even inside the myosin compartment (Fig. 2A). Myofibrillar irregularities are exemplified in Fig. 2A where sarcomeres are strongly off register and Z-lines show waviness. Occasionally, streaming Z-lines were observed in small cell regions (Fig. 2B). Their occurrence was rare but easily noticeable in whole tissue volume. Notably, the M-lines in the centre of A-bands were absent in slow $\mathrm{CK}^{-/-}$fibre. The SR network, abundant near A-bands in WT fibers (Fig. 1A), was scanty in $\mathrm{CK}^{-/-}$fibres (Fig. 1B, 2A,B). Sarcoplasmic reticulum as well as triads were noticeably less abundant especially around mitochondria, due to the irregular arrangement of myofibrils.
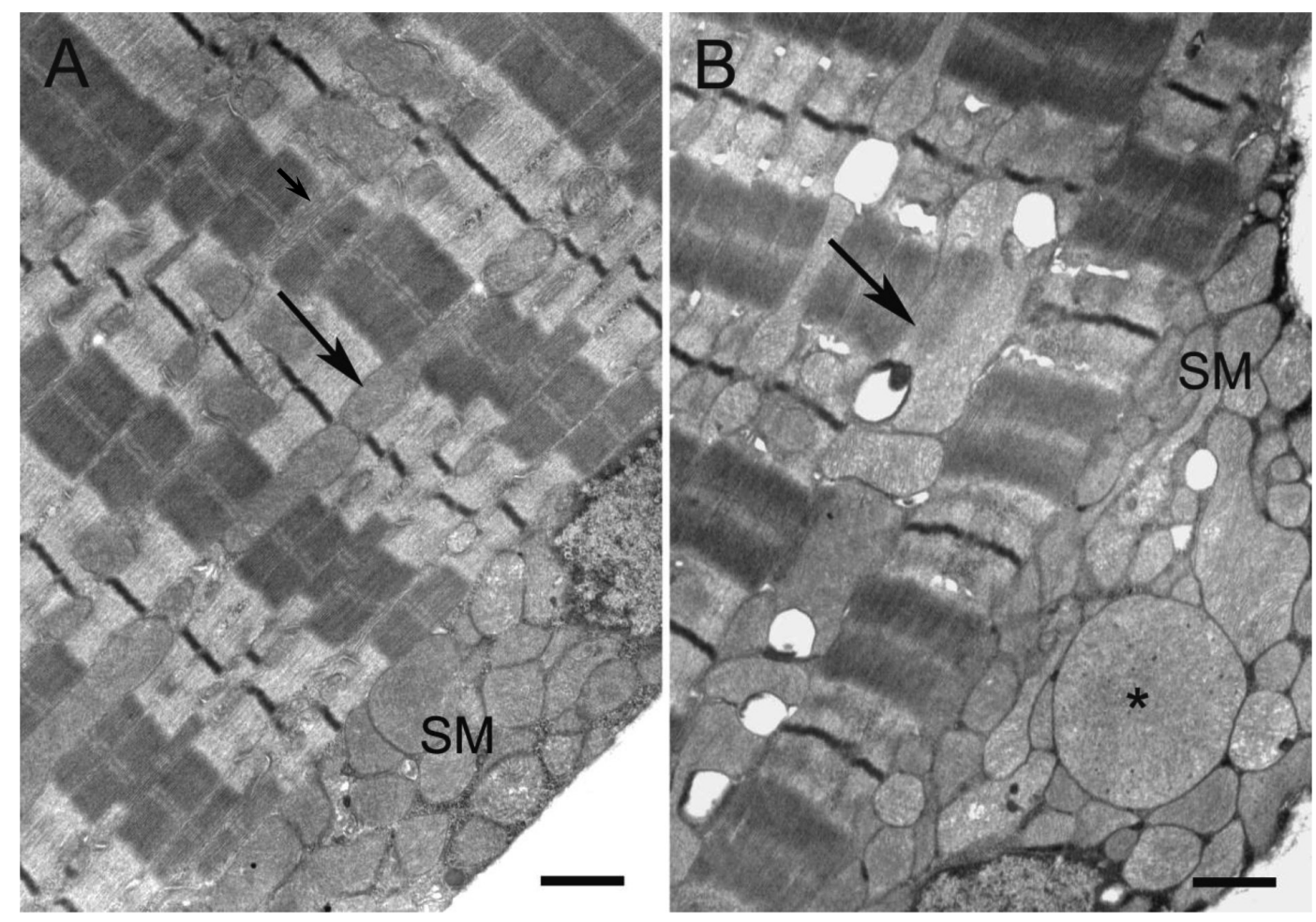

Figure 1. Electron micrographs of soleus muscle fibres of WT and $\mathrm{CK}^{-/-}$mice (longitudinal sections). A typical region of the soleus muscle fibre of WT mice (A) and $\mathrm{CK}^{-/-}$mice (B). SM, a subsarcolemmal cluster of mitochondria near the nucleus; arrow in panel A, the intermyofibrillar mitochondria; arrow in panel B, a large intermyofibrillar mitochondrion; small arrow, sarcoplasmic reticulum; asterisk, a large subsarcolemmal mitochondrion within the cluster of subsarcolemmal mitochondria (SM). Calibration bars $1 \mu \mathrm{m}$. 

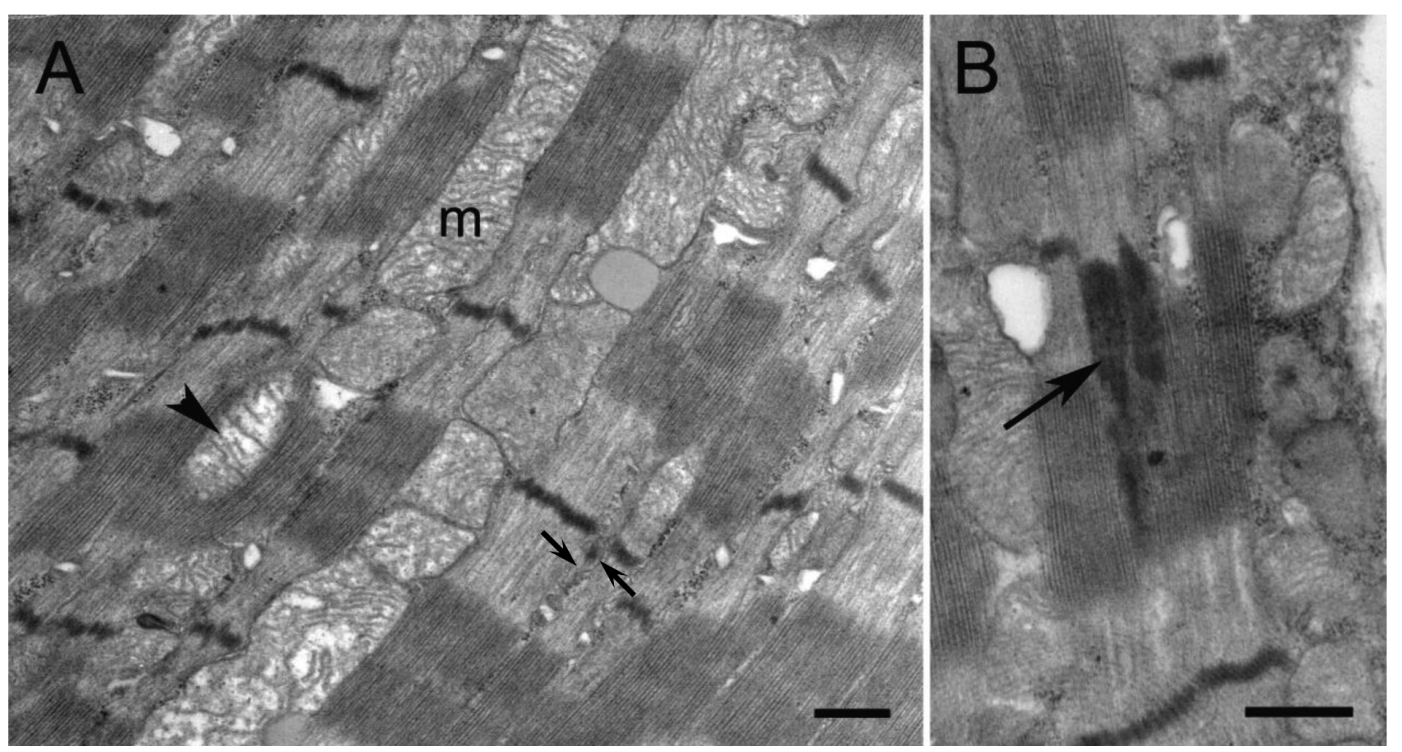

Figure 2. Details of soleus muscle fibres of $\mathrm{CK}^{-/-}$mice (longitudinal sections). A. A region with irregular arrangement of sarcomeres, wavy Z-lines, and intermyofibrillar mitochondria $(\mathrm{m})$ of variable shape and size. Arrowhead, a displaced mitochondrion in the centre of the A-band causing broadening of the sarcomere; small arrows, sarcoplasmic reticulum. B. A detail showing the streaming Z-lines (arrow). Calibration bars $500 \mathrm{~nm}$.

\section{Volume density and surface density analysis}

The average volume densities of organelles in slow skeletal muscle fibres of $\mathrm{CK}^{-/-}$and WT soleus are summarized in Table 1. As expected, myofibrils dominate the cell volume. Mitochondria and sarcoplasmic reticulum shared the remaining volume, living little room for cytosol.
The volume densities of organelles in $\mathrm{CK}^{-/}$fibres were different from those in WT. The volume densities of longitudinal sarcoplasmic reticulum, terminal cisterns and T-tubules in triads were significantly lower in $\mathrm{CK}^{-/-}$than in WT fibres. The volume density of mitochondria, especially of intermyofibrillar ones, was significantly higher in $\mathrm{CK}^{-/-}$than in WT fibres. The volume density of myofibrils in $\mathrm{CK}^{-/-}$fibres

Table 1. Volume and surface densities of different constituents of soleus muscle fibres of wild type (WT) and $\mathrm{CK}^{-/-}$mice

\begin{tabular}{|c|c|c|c|c|}
\hline & \multicolumn{2}{|c|}{ Volume densities (\%) } & \multicolumn{2}{|c|}{ Surface densities $\left(\mu \mathrm{m}^{-1}\right)$} \\
\hline & WT & $\mathrm{CK}^{-1-}$ & WT & $\mathrm{CK}^{-/-}$ \\
\hline subsarcolemmal mitochondria & $4.20 \pm 0.78$ & $6.67 \pm 0.79$ & $0.28 \pm 0.06$ & $0.37 \pm 0.10$ \\
\hline intermyofibrillar mitochondria & $12.79 \pm 1.02$ & $16.70 \pm 1.36^{*}$ & $1.48 \pm 0.07$ & $1.30 \pm 0.05$ \\
\hline total mitochondria & $16.99 \pm 1.71$ & $23.36 \pm 0.89^{*}$ & $1.76 \pm 0.11$ & $1.73 \pm 0.07$ \\
\hline Z-line & $3.95 \pm 0.36$ & $2.89 \pm 0.14^{\star}$ & $0.29 \pm 0.01$ & $0.16 \pm 0.02^{\star}$ \\
\hline I-band & $20.97 \pm 1.39$ & $28.05 \pm 1.10^{*}$ & $1.69 \pm 0.33$ & $1.14 \pm 0.09$ \\
\hline A-band & $35.68 \pm 2.09$ & $35.03 \pm 1.41$ & $1.03 \pm 0.07$ & $0.71 \pm 0.03^{*}$ \\
\hline total myofibrils & $60.59 \pm 1.56$ & $65.92 \pm 1.14^{\star}$ & $3.00 \pm 0.28$ & $2.00 \pm 0.13^{\star}$ \\
\hline sarcoplasmic reticulum & $14.61 \pm 1.65$ & $2.93 \pm 0.30^{*}$ & $3.92 \pm 0.26$ & $1.02 \pm 0.12^{\star}$ \\
\hline terminal cisterns of sarcoplasmic reticulum & $1.99 \pm 0.21$ & $1.26 \pm 0.11^{*}$ & $0.88 \pm 0.08$ & $0.40 \pm 0.07^{\star}$ \\
\hline transverse tubules & $1.26 \pm 0.05$ & $0.78 \pm 0.07^{\star}$ & $0.67 \pm 0.03$ & $0.34 \pm 0.03^{\star}$ \\
\hline sarcolemma & n.a. & n.a. & $0.01 \pm 0.01$ & $0.05 \pm 0.01^{\star}$ \\
\hline lipid drops & $0.52 \pm 0.26$ & $1.12 \pm 0.36$ & $0.04 \pm 0.02$ & $0.09 \pm 0.02$ \\
\hline cytosol & $4.14 \pm 0.86$ & $4.58 \pm 0.30$ & n.a. & n.a. \\
\hline
\end{tabular}

All values are presented as a mean \pm S.E. $(n=5)$. n.a., not applicable; ${ }^{\star} p<0.05$ compared with corresponding WT groups. 
exceeded slightly but significantly that in WT fibres but not in all myofibrillar segments. The volume density of I-bands increased, while the volume density of Z-lines decreased.

Surface density data (Table 1) indicate that in WT fibres the surface area of longitudinal sarcoplasmic reticulum was much larger than that of myofibrils and even more than of mitochondria. Differences in surface density data between the $\mathrm{CK}^{-/}$and WT fibres did not always follow the differences in volume density. In $\mathrm{CK}^{-/-}$fibres, surface densities of longitudinal sarcoplasmic reticulum, terminal cisterns and t-tubules were substantially less than that of WT fibres obviously but not exclusively due to a substantial reduction of their volume densities. Their surface-to-volume ratios indicate size reduction of sarcoplasmic reticulum but size increase of terminal cisterns and transverse tubules. The surface density of myofibrils also decreased, in spite of their higher volume density, due to their larger size in $\mathrm{CK}^{-/-}$fibres. The mitochondria did not change surface density and since they increased volume density, it is conceivable that they had to be larger than in WT fibres.

\section{Subcellular topology and environment of mitochondria}

The environment of mitochondria was evaluated for the subsarcolemmal and the intermyofibrillar mitochondria independently (Table 2). As the nuclei of the soleus muscle fibres are situated under the sarcolemma, perinuclear mitochondria were included into the subsarcolemmal mitochondria group.

In wild type fibres, the surface of subsarcolemmal mitochondria faced mostly the surface of other subsarcolemmal mitochondria, as expected for clusters, but contact with other organelles were not negligible (Table 2). The major nearest neighbour of intermyofibrillar mitochondria were membranes of the sarcoplasmic reticulum followed by Ibands of myofibrils.

In $\mathrm{CK}^{-/-}$fibres, the relative occupation of the subsarcolemmal mitochondria was substantially different from WT fibres. The communication area of subsarcolemmal mitochondria with membranes of longitudinal sarcoplasmic reticulum and with membranes of $t$-tubules was reduced substantially and that with membranes of terminal cisterns almost ceased. In addition, the occurrence of lipid droplets near subsarcolemmal mitochondria was particularly reduced. On the other hand, larger surface of subsarcolemmal mitochondria faced A-band.

The relative occupation of intermyofibrillar mitochondrial surface also changed substantially in $\mathrm{CK}^{-/-}$fibres (Table 2). Of high importance is much higher surface facing myofibrils, especially A-bands, cytosol and other intermyofibrillar mitochondria. On the other hand, the surface of intermyofibrillar mitochondria in the neighbourhood of the longitudinal sarcoplasmic reticulum was strongly reduced. The intermyofibrillar mitochondria changed their main association from sarcoplasmic reticulum to myofibrils.

\section{Environment of myofibrils}

The analysis of myofibrillar environment revealed substantial differences among constituents of myofibrils in wild-type fibres (Table 3). Z-lines were surrounded mostly by sarco-

Table 2. Environment of the subsarcolemmal (SM) and intermyofibrillar (IM) mitochondria of soleus muscle fibres of wild type (WT) and $\mathrm{CK}^{-/-}$mice

\begin{tabular}{|c|c|c|c|c|}
\hline & \multicolumn{4}{|c|}{ Environment (\%) } \\
\hline & SM WT & $\mathrm{SM} \mathrm{CK}^{-/-}$ & IM WT & $\mathrm{IM} \mathrm{CK}^{-/-}$ \\
\hline subsarcolemmal mitochondria & $38.00 \pm 7.28$ & $45.67 \pm 1.73$ & n.a. & $0.16 \pm 0.10$ \\
\hline intermyofibrillar mitochondria & $0.05 \pm 0.03$ & $0.42 \pm 0.20$ & $2.75 \pm 0.41$ & $10.92 \pm 3.06^{*}$ \\
\hline total mitochondria & $38.05 \pm 7.28$ & $46.09 \pm 1.92$ & $2.75 \pm 0.41$ & $11.08 \pm 3.09^{*}$ \\
\hline Z-line & $0.60 \pm 0.12$ & $0.37 \pm 0.11$ & $2.81 \pm 0.38$ & $1.92 \pm 0.37$ \\
\hline I-band & $3.49 \pm 0.61$ & $5.48 \pm 1.14$ & $24.93 \pm 4.27$ & $34.77 \pm 3.99$ \\
\hline A-band & $4.78 \pm 0.48$ & $6.63 \pm 0.59^{*}$ & $8.50 \pm 1.35$ & $23.04 \pm 1.37^{\star}$ \\
\hline total myofibrils & $8.87 \pm 0.90$ & $12.48 \pm 1.65$ & $36.24 \pm 3.89$ & $60.34 \pm 2.47^{\star}$ \\
\hline sarcoplasmic reticulum & $17.82 \pm 1.82$ & $2.44 \pm 0.67^{\star}$ & $40.42 \pm 3.42$ & $7.39 \pm 0.28^{*}$ \\
\hline terminal cisterns of sarcoplasmic reticulum & $4.59 \pm 0.92$ & $0.17 \pm 0.07^{\star}$ & $8.85 \pm 0.95$ & $1.99 \pm 0.36^{*}$ \\
\hline transverse tubules & $4.41 \pm 0.89$ & $1.15 \pm 0.50^{*}$ & $5.74 \pm 0.74$ & $0.82 \pm 0.18^{*}$ \\
\hline sarcolemma & $1.87 \pm 0.62$ & $6.04 \pm 1.27^{\star}$ & $0.01 \pm 0.01$ & n.a. \\
\hline lipid drops & $8.01 \pm 3.59$ & $1.14 \pm 0.34^{\star}$ & $2.71 \pm 1.10$ & $2.43 \pm 0.58$ \\
\hline cytosol & $16.38 \pm 9.28$ & $30.49 \pm 1.93$ & $3.08 \pm 1.11$ & $16.56 \pm 1.60^{\star}$ \\
\hline
\end{tabular}

All values are presented as a mean \pm S.E. $(n=5)$. n.a., not applicable; ${ }^{\star} p<0.05$ compared with corresponding WT groups. 
Table 3. Environment of the myofibrils and their constituents of soleus muscle fibres of wild type (WT) and $\mathrm{CK}^{-/-}$mice

\begin{tabular}{|c|c|c|c|}
\hline & & \multicolumn{2}{|c|}{ Environment (\%) } \\
\hline & & WT & $\mathrm{CK}^{-/-}$ \\
\hline \multirow{8}{*}{ A-band } & total mitochondria & $19.37 \pm 2.10$ & $32.08 \pm 2.79^{*}$ \\
\hline & Z-line & $0.06 \pm 0.03$ & $0.44 \pm 0.24$ \\
\hline & I-band & $4.64 \pm 0.86$ & $11.09 \pm 0.71^{\star}$ \\
\hline & sarcoplasmic reticulum & $46.97 \pm 1.89$ & $34.80 \pm 2.46^{*}$ \\
\hline & terminal cisterns of sarcoplasmic reticulum & $2.51 \pm 0.83$ & $1.53 \pm 0.59$ \\
\hline & transverse tubules & $5.22 \pm 2.17$ & $2.84 \pm 0.32$ \\
\hline & lipid drops & $0.90 \pm 0.42$ & $2.68 \pm 1.01$ \\
\hline & cytosol & $20.34 \pm 1.38$ & $14.55 \pm 0.76^{*}$ \\
\hline \multirow{8}{*}{ I-band } & total mitochondria & $42.47 \pm 1.63$ & $33.12 \pm 3.13^{\star}$ \\
\hline & Z-line & $1.18 \pm 0.17$ & $2.76 \pm 0.65^{\star}$ \\
\hline & A-band & $3.47 \pm 0.35$ & $7.49 \pm 0.68^{\star}$ \\
\hline & sarcoplasmic reticulum & $17.18 \pm 1.18$ & $17.14 \pm 1.68$ \\
\hline & terminal cisterns of sarcoplasmic reticulum & $7.59 \pm 0.86$ & $6.57 \pm 1.44$ \\
\hline & transverse tubules & $8.94 \pm 0.60$ & $10.93 \pm 1.13$ \\
\hline & lipid drops & $0.67 \pm 0.26$ & $1.91 \pm 0.61$ \\
\hline & cytosol & $18.51 \pm 1.24$ & $20.07 \pm 1.03$ \\
\hline \multirow{8}{*}{ Z-line } & total mitochondria & $20.12 \pm 2.38$ & $20.48 \pm 1.10$ \\
\hline & I-band & $6.02 \pm 1.66$ & $15.89 \pm 3.51^{\star}$ \\
\hline & A-band & $0.23 \pm 0.16$ & $1.76 \pm 0.98$ \\
\hline & sarcoplasmic reticulum & $49.56 \pm 1.65$ & $40.94 \pm 3.99$ \\
\hline & terminal cisterns of sarcoplasmic reticulum & 0.0 & $0.09 \pm 0.09$ \\
\hline & transverse tubules & $0.07 \pm 0.07$ & $0.09 \pm 0.09$ \\
\hline & lipid drops & $0.39 \pm 0.19$ & $1.10 \pm 0.41$ \\
\hline & cytosol & $23.61 \pm 1.83$ & $19.65 \pm 1.68$ \\
\hline
\end{tabular}

plasmic reticulum. I-bands were occupied preferentially by mitochondria, less by sarcoplasmic reticulum and cytosol. The A-bands were largely covered by sarcoplasmic reticulum, thereafter by mitochondria and cytosol. From the viewpoint of organelles, one can see that mitochondria covered myofibrils mostly at I-bands. Sarcoplasmic reticulum covered preferentially Z-lines and A-bands. Triadic complexes contacted myofibrils at I-bands close to I/A edge. Lipid droplets were spread scantily and at random.

In $\mathrm{CK}^{-/-}$fibres, the environment of myofibrils was more even than in WT fibres. Important factor was the reduced positional register of sarcomeres of neighbour myofibrils. Moreover, the dominance of sarcoplasmic reticulum diminished due to the reduced sarcoplasmic reticulum and increased mitochondrial fractions. Viewed from the point of organelles, mitochondria covered A- and I-bands to similar extent but sarcoplasmic reticulum still covered preferentially A-bands and Z-lines. Complexes of terminal cisterns and $\mathrm{t}$-tubules were still at I-bands but with decreased overlap to A-bands. Interestingly, the fraction of cytosol changed only near A-bands, where it was significantly lower in $\mathrm{CK}^{-/-}$than in WT fibres.

\section{Comparison of the slow and fast twitch muscle fibres}

For comparison with the fast twitch muscle fibres, we have used a dataset on mouse gastrocnemius muscle published previously (Novotova et al. 2006). Per unit volume, the slow twitch fibres contain three times more mitochondria, two times more sarcoplasmic reticulum and by $27 \%$ less myofibrils than the fast fibres. These differences are in line with functional or physiological characteristics of the soleus and the gastrocnemius muscles. For instance, in slow twitch fibres the volume density ratio of mitochondria to myofibrils was $4 \times$ higher than in fast twitch fibres $(0.28 v s$. 0.07$)$. The volume density ratio of mitochondria to sarcoplasmic reticulum and terminal cisterns was also substantially higher in soleus fibres $(1.7 \times)$. The relative content of terminal cisterns was similar in both muscle types but their average size, estimated as a surface-to-volume ratio, was by $30 \%$ smaller in soleus. 
The relative content of $t$-tubules was much higher $(2.5 \times)$ in soleus than in gastrocnemius but not due to size, which was almost the same. The myofibrils were more abundant in gastrocnemius fibres but their support by mitochondria and sarcoplasmic reticulum was much less developed. Mitochondria were very different between the slow and the fast myofibres. In slow twitch fibres, the subsarcolemmal mitochondria were $4 \times$ more abundant and had more than $5 \times$ larger surface area. The intermyofibrillar mitochondria were $3 \times$ more abundant according the volume as well as the surface densities. The environment analysis of slow fibres indicated that subsarcolemmal mitochondria formed more clusters, more contacts with sarcoplasmic reticulum and triads, but much less contacts with myofibrils and sarcolemma. Intermyofibrillar mitochondria preferred sarcoplasmic reticulum in slow but myofibrils in fast fibres. They approach the terminal cisterns to the same extent in both fibre types but the transverse tubules by an order of magnitude less in fast fibres. Free cytosol was much less abundant around mitochondria, especially the interfibrillar ones, in slow fibres. Lipid droplets were marginal in fast fibres but they occupied significant portion of mitochondrial surface in slow fibres. Intermyofibrillar mitochondria preferred I-band to A-band in both fibre types but more pronouncedly so in fast fibres. However, the subsarcolemmal mitochondria do not have a special organelle preference in either fibre type.

\section{Discussion}

This study provides two sets of data. First, the quantitative description of the ultrastructure of soleus muscle fibres, which represent an epitome of the oxidative slow-twitch type of mammalian skeletal muscle fibres. Second, the quantitative description of changes in the ultrastructure that stem from the lack of creatine kinase, a key enzyme in energetics of this muscle. The both data sets are based on the environment of organelles, the measure that we introduced as a sensitive indicator of the cell composition and organization (Novotova et al. 2006). Quantitative data on structural composition of slow muscle fibres are sparse in the literature. The ultrastructure of slow skeletal muscle fibres of mouse was, to our best knowledge, described only by Ogata (1964).

\section{Effects of CK deficiency on architecture of slow muscle}

Changes in the ultrastructure of skeletal muscle fibres following invalidation of cytosolic and mitochondrial creatine kinase were described first by van Deursen et al. (1993) and Steeghs et al. (1997, 1998), without details on slow fibre types. The general morphological changes involved overall increase in mitochondrial volume, increased number of lipid droplets, and no apparent changes in the ultrastructure of sarcomeres. Our study extends this description in many details and provides a new view based on quantification of ultrastructural changes.

The principal effects of invalidation of CK system on composition of slow twitch fibres were as follows:

1) The volume density of mitochondrial populations substantially increased due to increased size of mitochondria.

2) The volume density of sarcoplasmic reticulum sharply decreased together with its surface density. Reduction of sarcoplasmic reticulum near mitochondria and A-bands was specific for slow fibres as no similar changes were observed in fast fibres.

3) Relative content of terminal cisterns and of t-tubules was reduced, albeit less than their surface what indicates increase in their size.

4) Contractile myofibrils gained in volume density at reduced surface-to-volume ratio suggesting increased diameter of myofibrils and, in effect, increased diffusion distances for ATP and calcium during the contractionrelaxation cycle. This effect was opposite to fast fibres.

5) The relative surface of mitochondria occupied by myofibrils was increased in $\mathrm{CK}^{-/-}$fibres, meaning that larger portion of energy produced by mitochondria might be transferred directly to myofibrils. A similar reaction was observed in the fast fibres.

6) In WT fibres mitochondria support preferentially calcium cycling, while in $\mathrm{CK}^{-/-}$fibres they support preferentially contractility. This is very different from the fast fibres where such cross-switch was not observed.

7) In WT fibres, membranes of sarcoplasmic reticulum and free cytosol dominated near A-bands, but they were by a large part replaced by mitochondria in $\mathrm{CK}^{-/-}$fibres. This cross-switch indicates impaired structural support of calcium cycling but enhanced structural support to energy supply, a reasonable response to constrained energy transfer capacity. I-bands of $\mathrm{CK}^{-/-}$fibres displayed reduced mitochondrial support and unchanged support of calcium cycling.

These results clearly demonstrate the character and the extent of the cytoarchitectural remodelling towards increased oxidative capacity and increased interaction between myofibrils and mitochondria even at the expense of reduced role of sarcoplasmic reticulum in calcium handling.

In slow muscles of ${\mathrm{M}-\mathrm{CK}^{-/-}}^{-}$and of $\mathrm{CK}^{-/-}$mice, van Deursen et al. (1993) and Steeghs et al. (1997) reported a 30\% increase in mitochondria situated between myofibrils. The present study revealed that also the volume of subsarcolemmal mitochondria was substantially increased. Interestingly, a preferential increase in subsarcolemmal, compared to intermyofibrillar mitochondria, was observed in skeletal 
muscle in response to endurance training (Hoppeler et al. 1985; Hoppeler and Fluck 2003). Mitochondrial transcripts showed a 1.2 to 2.5 -fold increase in the signal intensity in $\mathrm{CK}^{-/-}$soleus muscle (de Groof et al 2001b). Similarly, quantitative Western-blot analysis of $\mathrm{CK}^{-/-}$soleus muscle also evidenced a significant increase in some mitochondrial proteins (de Groof et al. 2001a) like adenine nucleotide translocase, cytochrome oxidase and phosphate carrier. $\mathrm{LDH}$ isoenzyme determination also showed a shift towards a more oxidative profile consistent with earlier report (de Groof et al. 2001a). Taken together these results show that in response to CK deficiency, mitochondrial volume and activity of slow fibres are also increased although to a lesser extent than in fast skeletal muscles (Steeghs et al. 1997; Kaasik et al. 2001; Novotova et al. 2006). It has been speculated that this increase in oxidative capacity of skeletal muscle is an adaptive response to the lack of phosphotransfer system induced by creatine kinase deficiency (Bruton et al. 2003). It was also recognized that the nature and magnitude of the compensatory adaptations in muscles of CK deficient mice are strongly cell-type dependent (de Groof et al. 2001b).

The quantitative analysis of mitochondrial environment, revealed different features of cellular adaptations in slow soleus fibres than in fast gastrocnemius fibres. The large increase in the contact area of subsarcolemmal mitochondria with A-bands and sarcolemma together with insignificant change in mutual subsarcolemmal mitochondrial contacts indicates that the increased volume density of mitochondria under sarcolemma was accompanied by their spreading rather than by their grouping beneath the sarcolemma. Additionally, the increased proximity of mitochondria with A-bands in slow $\mathrm{CK}^{-/-}$fibres resulted also from decreased amounts of sarcoplasmic reticulum near myofibrils. To a certain extent, this effect might also result from intermyofibrillar mitochondrial invasion of myofibrils (Figure 2A), as was described already in cardiac myocytes of $\mathrm{CK}^{-/-}$mice (Kaasik et al. 2001). The creatine kinase shuttle of slow muscles had been shown to be important during exercise for more efficient coupling between energy production and utilization (Zoll et al. 2002; Perry et al. 2012). Despite morphological and biochemical remodelling, exercise capacity of $\mathrm{CK}^{-/-}$mice was reduced (Momken et al. 2005).

The reduction of sarcoplasmic reticulum between mitochondria and A-bands is an example of specific and effective rearrangement of intracellular structures to compensate for impaired flow of energy. This exemplifies an adaptive process that increases direct supply of ATP to contractile machinery and, at the same time, decreases local ATP consumption by sarcoplasmic reticulum. It differs principally from the adaptation observed in the fast $\mathrm{CK}^{-/-}$fibres of gastrocnemius where the mitochondrial volume was also augmented, but the relocation of mitochondria towards the A-bands, virtually free of mitochondria in wild types, was dominant
(Novotova et al. 2006). Functionally this was accompanied by an increase in the direct coupling between mitochondria and myofibrils (Kaasik et al. 2003). It was speculated that these functional changes were aimed at rescuing the local control of adenine nucleotides lost by $\mathrm{CK}$ ablation. In this concept, direct spatial interactions of organelles involved in energy fluxes allow to maintain a high ATP/ADP ratio near ATPases.

It might be speculated that mitochondria that replaced SR near A-bands participate in relaxation of contraction. Indeed, mitochondrial $\mathrm{Ca}^{2+}$ concentration (measured with rhod-2 and confocal microscopy) increased during repeated tetanic stimulation in mitochondria of $\mathrm{CK}^{-/-}$but not of wild-type flexor digitorum brevis fibres (Bruton et al. 2003). However, inhibition of mitochondria in $\mathrm{CK}^{-/-}$soleus, although strongly affecting the tetanic force, did not affect intracellular calcium changes, suggesting that calcium regulation is not primarily supported by mitochondrial ATP production (Bruton et al. 2003). This study suggested that triadic regions could have a high capacity to generate ATP via anaerobic glycolysis. In support of their hypothesis, they reported that this region contains a high concentration of glycolytic enzymes and the glycolysis supported calcium uptake was demonstrated (Xu et al. 1995). It is of interest that the glycolytic as well as the oxidative capacity was increased in skeletal muscle of $\mathrm{CK}^{-/-}$ mice (Steeghs et al. 1998), and that the glycolytic support of sarcoplasmic reticulum calcium uptake was indeed increased in $\mathrm{CK}^{-/-}$cardiac fibres (Boehm et al. 2000). Thus it can be proposed that the glycolytic ATP production would be more directed to sarcoplasmic reticulum while the oxidative ATP production would support primarily the contractile function. Thus, as suggested by Bruton et al. (2003), this may be explained by an increased reliance of the triadic region on bound glycolytic enzymes.

To summarize, this study on the slow oxidative skeletal muscle fibres provided strong support to our previous conclusion drawn in an analogous study on the fast glycolytic skeletal muscle fibres (Novotova et al. 2006), namely that the architecture of muscle cells is under tight control of a cellular program that optimizes efficiency of energy utilization. Now we may add that this program is specific to each principal muscle cell type. This finding explains the large variability observed in adaptation of various muscle groups to changes in the workload or in other physiological or pathological conditions.

Quantification of the architectural features, such as the environment of organelles, may be very useful in considerations on functional phenotype of different muscles. However, the relations among structural and functional features are not trivial. Additionally, the architecture of muscle cells is by its nature very complex and difficult to describe and depict. Therefore, the best use of these data can be in construction of authentic mathematical and/or geometrical models ( $\mathrm{Pa}-$ rulek et al. 2007). 
Acknowledgements. The study was supported by VEGA 2/0110/15, 2/0147/14 and APVV-15-0302 grants. Renée VenturaClapier is emeritus scientist at CNRS.

Conflict of interest. The authors declare no conflicts of interests.

\section{References}

Baddeley A. J., Gundersen H. J. G., Cruz-orive L. M. (1986): Estimation of surface area from vertical sections. J. Microsc. 142, 259-276 http://dx.doi.org/10.1111/j.1365-2818.1986.tb04282.x

Boehm E., Ventura-Clapier R., Mateo P., Lechene P., Veksler V. (2000): Glycolysis supports calcium uptake by the sarcoplasmic reticulum in skinned ventricular fibres of mice deficient in mitochondrial and cytosolic creatine kinase. J. Mol. Cell. Cardiol. 32, 891-902 http://dx.doi.org/10.1006/jmcc.2000.1130

Bruton J. D., Dahlstedt A. J, Abbate F., Westerblad H. (2003): Mitochondrial function in intact skeletal muscle fibres of creatine kinase deficient mice. J. Physiol. 552, 393-402 http://dx.doi.org/10.1113/jphysiol.2003.050732

de Groof A. J., Oerlemans F. T., Jost C. R., Wieringa B. (2001a): Changes in glycolytic network and mitochondrial design in creatine kinase-deficient muscles. Muscle Nerve 24, 1188-1196 http://dx.doi.org/10.1002/mus.1131

de Groof A. J., Smeets B., Groot Koerkamp M. J., Mul A. N., Janssen E. E., Tabak H. F., Wieringa B. (2001b): Changes in mRNA expression profile underlie phenotypic adaptations in creatine kinase-deficient muscles. FEBS Lett. 506, 73-78 http://dx.doi.org/10.1016/S0014-5793(01)02879-4

Hoppeler H., Howald H., Conley K., Lindstedt S. L., Claassen H., Vock P., Weibel E. R. (1985): Endurance training in humans: aerobic capacity and structure of skeletal muscle. J. Appl. Physiol. 59, 320-327

Hoppeler H., Fluck M. (2003): Plasticity of skeletal muscle mitochondria: structure and function. Med. Sci. Sports Exerc. 35, 95-104 http://dx.doi.org/10.1097/00005768-200301000-00016

Kaasik A., Veksler V., Boehm E., Novotova M., Minajeva A., Ventura-Clapier R. (2001): Energetic crosstalk between organelles: architectural integration of energy production and utilization. Circ. Res. 89, 153-159 http://dx.doi.org/10.1161/hh1401.093440

Kaasik A., Veksler V., Boehm E., Novotova M., Minajeva A., Ventura-Clapier R. (2003): From energy store to energy flux: a study in creatin kinase deficient fast skeletal muscle. FASEB J. 17, 708-710 http://dx.doi.org/10.1096/fj.02-0684fje

Karen P., Kubinova L., Krekule I. (1998): STESYS software for computerassisted stereology. Physiol. Res. 47, 271-278

Meyer R. A., Sweeney H. L., Kushmerick M. J. (1984): A simple analysis of the „phosphocreatine shuttle“. Am. J. Physiol. Cell Physiol. 246, C365-377

Momken, I., Lechene P., Koulmann N., Fortin D., Mateo P., Doan B.T., Hoerter J., Bigard X., Veksler V., Ventura-Clapier R. (2005): Impaired voluntary running capacity of creatine kinasedeficient mice. J. Physiol. 565, 951-964 http://dx.doi.org/10.1113/jphysiol.2005.086397

Novotova M., Pavlovicova M,. Veksler V. I., Ventura-Clapier R., Zahradnik I. (2006): Ultrastructural remodeling of fast skeletal muscle fibers induced by invalidation of creatine kinase. Am. J. Physiol. Cell Physiol. 291, 1279-1285 http://dx.doi.org/10.1152/ajpcell.00114.2006

Ogata T. (1964): An electron microscopic study on red, white and intermediate muscle fiber of mouse. Acta Med. Okayama 18, 271-280

Parulek J., Ciglan M., Simon B., Sramek M., Hluchy L., Zahradnik I. (2007): Grid Problem Solving Environment for Stereology Based Modeling. International Conference on Grid computing, High-performance and Distributed Applications, OTM 2007, Part II, LNCS 4804, pp. 1417-1434, Springer-Verlag Berlin Heidelberg http://dx.doi.org/10.1007/978-3-540-76843-2_20

Perry C. G., Kane D. A., Herbst E. A., Mukai K., Lark D. S., Wright D. C., Heigenhauser G. J., Neufer P. D., Spriet L. L., Holloway G. P. (2012): Mitochondrial creatine kinase activity and phosphate shuttling are acutely regulated by exercise in human skeletal muscle. J. Physiol. 590, 5475-5486 http://dx.doi.org/10.1113/jphysiol.2012.234682

Saks V. A., Khuchua Z. A., Vasilyeva E. V., Belikova O. Y., Kuznetsov A. V. (1994): Metabolic compartmentation and substrate channelling in muscle cells. Role of coupled creatine kinases in in vivo regulation of cellular respiration - a synthesis. Mol. Cell. Biochem. 133, 155-192 http://dx.doi.org/10.1007/BF01267954

Steeghs K., Benders A., Oerlemans F., deHaan A., Heerschap A., Ruitenbeek W., Jost C., van Deursen J., Perryman B., Pette D. et al. (1997): Altered Ca2+ responses in muscles with combined mitochondrial and cytosolic creatine kinase deficiencies. Cell 89, 93-103 http://dx.doi.org/10.1016/S0092-8674(00)80186-5

Steeghs K., Oerlemans F., de Haan A., Heerschap A., Verdoodt L., de Bie M., Ruitenbeek W., Benders A., Jost C., van Deursen J. et al. (1998): Cytoarchitectural and metabolic adaptations in muscles with mitochondrial and cytosolic creatine kinase deficiencies. Mol. Cell. Biochem. 184, 183-194 http://dx.doi.org/10.1023/A:1006811717709

van Deursen J., Heerschap A., Oerlemans F., Ruitenbeek W., Jap P., ter Laak H., Wieringa B. (1993): Skeletal muscles of mice deficient in muscle creatine kinase lack burst activity. Cell 74, 621-631 http://dx.doi.org/10.1016/0092-8674(93)90510-W

Veksler V. I., Kuznetsov A. V., Anflous K., Mateo P., van Deursen J., Wieringa B., Ventura-Clapier R. (1995): Muscle creatine kinase-deficient mice.2. Cardiac and skeletal muscles exhibit tissue-specific adaptation of the mitochondrial function. J. Biol. Chem. 270, 19921-19929 http://dx.doi.org/10.1074/jbc.270.34.19921

Ventura-Clapier R., Kuznetsov A. V., d'Albis A., van Deursen J., Wieringa B., Veksler V. I. (1995): Muscle creatine kinasedeficient mice.1. Alterations in myofibrillar function. J. Biol. Chem. 270, 19914-19920 http://dx.doi.org/10.1074/jbc.270.34.19914

Watchko J. F., Daood M. J., Sieck G. C., LaBella J. J., Ameredes B. T., Koretsky A. P., Wieringa B. (1997): Combined myofibrillar 
and mitochondrial creatine kinase deficiency impairs mouse diaphragm isotonic function. J. Applied Physiol. 82, 1416-1423

Wyss M., Smeitink J., Wevers R. A., Wallimann T. (1992): Mitochondrial creatine kinase - a key enzyme of aerobic energy metabolism. Biochim. Biophys. Acta 1102, 119-166 http://dx.doi.org/10.1016/0005-2728(92)90096-K

Xu K. Y., Zweier J. L., Becker L. C. (1995): Functional coupling between glycolysis and sarcoplasmic reticulum $\mathrm{Ca} 2+$ transport. Circ. Res. 77, 88-97 http://dx.doi.org/10.1161/01.RES.77.1.88

Zoll J., Sanchez H., N'Guessan B., Ribera F., Lampert E., Bigard X., Serrurier B., Fortin D., Geny B., Veksler V., Ventura-Clapier R.,
Mattauer B. (2002): Physical activity changes the regulation of mitochondrial respiration in human skeletal muscle. J. Physiol. 543, 191-200

http://dx.doi.org/10.1113/jphysiol.2002.019661

Žurmanová J., Soukup T. (2013): Comparison of myosin heavy chain mRNAs, protein isoforms and fiber type proportions in the rat slow and fast muscles. Physiol. Res. 62, 445-453

Received: June 8, 2016

Final version accepted: September 1, 2016

First published online: September 9, 2016 